\title{
Analytical Model of Semiconductor Sensor Layers in SAW Gas Sensors
}

\author{
T. HEJCZYK ${ }^{a}$, M. URBAŃCZYK ${ }^{b}$ AND W.P. JAKUBIK ${ }^{c}$ \\ ${ }^{a}$ ENTE Sp. z o.o., Gaudiego 7, 44-100 Gliwice, Poland \\ ${ }^{b}$ Faculty of Electrical Engineering, Silesian University of Technology, Krzywoustego 2, 44-100 Gliwice, Poland \\ ${ }^{c}$ Institute of Physics, Silesian University of Technology, Krzywoustego 2, 44-100 Gliwice, Poland \\ In the paper a new theoretical model for analyzing a surface acoustic wave gas sensor is presented. Basing on \\ the electric load of the piezoelectric acoustic line the effect of surface acoustic wave velocity changes vs. surface \\ conductivity is predicted which depends on the profile concentration of gas molecules diffused into the porous film. \\ Inside the sensor layer Knudsen's model of gas diffusion was used.
}

PACS numbers: 77.65.Dq, 68.35.Iv, 07.07.Df

\section{Introduction}

The aim of the investigations was to study thin film interaction with target gases in the SAW sensor configuration. Thin film layers have different properties and characteristics than the conventional bulk substrate [1-3]. The paper summarizes the acoustoelectric theory, i.e. Ingebrigtsen's formula [4], impedance transformation law, gas concentration profiles, and predicts the influence of a thin semiconductor sensor layer with Knudsen's gas diffusion model on the SAW wave velocity in a piezoelectric acoustic waveguide. In the paper a new theory for analyzing SAW gas sensor is proposed. Main setup of a bilayer sensor for hydrogen detection is shown in Fig. 1.

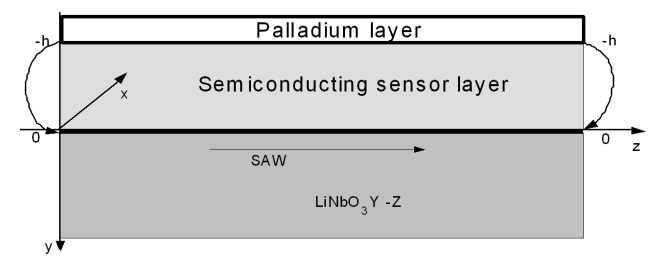

Fig. 1. Model of the layered SAW gas sensor - thin palladium film on the semiconducting sensor layer (in hydrogen gas sensor).

It consists of a thin palladium film as catalyst for hydrogen molecules and a semiconducting sensor layer (metal oxide or a polymeric one). Optimizing this structure, we can obtain a high sensitivity of hydrogen detection. This is very important in the application of other gas sensors. We must determine the thickness of the layer and the temperature of operation, and other parameters to reach a high sensitivity. By proper design, using a bilayer structure consisting sublayers, the goal is to optimize a hydrogen SAW sensor as shown in Fig. 1.

In Fig. 1 for $y<0$ we assume the isotropic conditions so that $\varepsilon$ is a scalar quantity with the value $\varepsilon_{0}$. Be- cause the piezoelectric medium is anisotropic, we express the dielectric permittivity in the region $y>0$ in tensor form. The appropriate value of $\varepsilon$ is approximately the stress-free form $\varepsilon^{\mathrm{T}}$, where $\varepsilon_{p}^{\mathrm{T}}=\sqrt{\varepsilon_{y y}^{\mathrm{T}} \varepsilon_{z z}^{\mathrm{T}}-\left(\varepsilon_{y z}^{\mathrm{T}}\right)^{2}}$. The piezoelectric medium (at $y>0$ ) and imaginary charge sheet $($ at $y<0)$ are in three regions in $y$ directions [4].

The perturbed boundary conditions will be specified in terms of the normalized surface impedance $z_{E}^{\prime}(0)[4,5]$. Let us assume that the surface conductivity is independent of the frequency of the acoustic wave and the surface impedance $z_{E}^{\prime}(0)$ is only a function of the surface conductivity, $\sigma_{\mathrm{s}}$, and the SAW velocity of propagation, $v_{0}$

$$
z_{E}^{\prime}(0)=\left(-\mathrm{i}+\frac{\sigma_{\mathrm{s}}}{\varepsilon_{0} v_{0}}\right)^{-1}
$$

where $\sigma_{\mathrm{s}}=\sigma h, \sigma$ - bulk conductivity, $h$ - thickness of semiconductor layer.

\section{Gas concentration profile in the sensing layer}

The mechanism of gas diffusion through a porous material depends on the size of pores and the type of surface diffusion: Knudsen's and a molecular one. In this paper we are focusing on the Knudsen diffusion which depends on the pore radius and prevails in the pores ranging from 1 to $100 \mathrm{~nm}$ in radius [1]. In this way the Knudsen diffusion constant, $D_{\mathrm{K}}$, is defined to describe these phenomena. This constant $D_{\mathrm{K}}$ depends on the molecular weight of the diffusing gas, $M$, the pore radius, $r$, temperature, $T$, and the universal gas constant, $R$, as follows:

$$
D_{\mathrm{K}}=\frac{4 r}{3} \sqrt{\frac{2 R T}{\pi M}} .
$$

We assume that $r$ is in the pore size range, i.e. $r>2 \mathrm{~nm}$ in order to reduce the size of the pores to uniform pores with a representative radius $r$. In this environment exists a free molecular flow. This means that gas molecules collide more frequently with the boundaries than with other gas molecules. The original studies of a free-molecule 
flow were limited to small holes in very thin plates. This ensured that the molecules did not collide with each other while passing through the hole, and hence that the molecules moved entirely independently of each other.

Gas molecules are consumed rapidly or slowly due to the surface reaction in the sensing layer [1]. We consider the gas diffusion into a porous thin semiconducting film which is exposed to a target gas, as shown in Fig. 2.

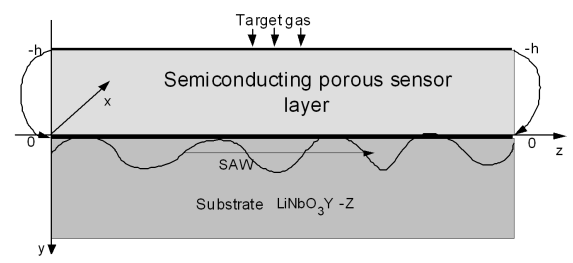

Fig. 2. Model of a gas semiconducting sensor layer.

Two assumptions, i.e. the Knudsen diffusion and first-order surface reaction, allow us to formulate the well-known diffusion equation $[1,6]$ :

$$
\frac{\partial C_{\mathrm{A}}}{\partial t}=D_{\mathrm{K}} \frac{\partial^{2} C_{\mathrm{A}}}{\partial x^{2}}-k C_{\mathrm{A}},
$$

where $C_{\mathrm{A}}$ is the concentration of target gas, $t$ - time, $D_{\mathrm{K}}$ - the Knudsen diffusion constant, $y$ - distance from the bottom layer, counted from the piezoelectric substrate, $k$ is the rate constant.

At the steady-state derivative

$$
\frac{\partial C_{\mathrm{A}}}{\partial t}=0 \text {. }
$$

Equation (1) is modified into the formula $[1,6]$ :

$$
D_{\mathrm{K}} \frac{\partial^{2} C_{\mathrm{A}}}{\partial y^{2}}-k C_{\mathrm{A}}=0
$$

The general solution of this equation is expressed as

$$
C_{\mathrm{A}}=C_{1} \exp \left(y \sqrt{\frac{k}{D_{\mathrm{K}}}}\right)+C_{2} \exp \left(-y \sqrt{\frac{k}{D_{\mathrm{K}}}}\right),
$$

here $C_{1}$ and $C_{2}$ are integral constants.

The boundary conditions at surface $(y=-h)$ are

$$
C_{\mathrm{A}}=C_{\mathrm{A}, \mathrm{s}}, \quad \frac{\partial C}{\partial y}=0 .
$$

The conditions quoted above allow us to determine integral constants.

The following equation can be obtained $[1,7]$ :

$$
C_{\mathrm{A}}=C_{\mathrm{A}, \mathrm{s}} \frac{\cosh \left(|y| \sqrt{k / D_{\mathrm{K}}}\right)}{\cosh \left(|-h| \sqrt{k / D_{\mathrm{K}}}\right)} .
$$

In Eq. (8) $C_{\mathrm{A}, \mathrm{s}}$ is the target gas concentration outside the film at the surface $y=-h$. We see that the concentration profile depends on the thickness of the sensor layer and the constants $k$ and $D_{\mathrm{K}}$.

Obviously the profiles change sharply with changing $\sqrt{k / D_{\mathrm{K}}}$.

As we see, the gas concentration inside the film is not constant. The gas concentration decreases with the depth due to the surface reactions diffusion phenomena.
A main assumption is that the electrical conductance $\sigma(y)$ of the sheet under exposure to the target gas is linear to the gas concentration $\left(C_{\mathrm{A}}\right)[1]$ :

$$
\sigma(y)=\sigma_{0}\left(1 \pm a C_{\mathrm{A}}\right),
$$

where $\sigma_{0}$ is the layer conductance in air, $a$ is the coefficient defined as the sensitivity coefficient. Experimental data for $\mathrm{H}_{2}$ have been presented in [1], when $C_{\mathrm{A}, \mathrm{s}}$ was fixed at $800 \mathrm{ppm}$ at a temperature of $350{ }^{\circ} \mathrm{C}$, and assumed to be $a=1 \mathrm{ppm}^{-1}$. For these data the sensitivity data for $\mathrm{H}_{2}$ fit fairly well to the correlation line for $\sqrt{k / D_{\mathrm{K}}}=0.01 \mathrm{~nm}^{-1}$.

The electrical conductance of the whole film is given by integrating $\sigma(y)$ over the whole range of $y(y=-h ;-0)$. This treatment has been proposed by Sakai, Williams and Hilger $[1,8,9]$. We cannot treat a semiconductor layer in a homogeneous way of thinking, because the profile of molecule gas concentration in a semiconducting sensor layer changes with the distance from the piezoelectric substrate (Fig. 3) and may influence the acoustoelectric interaction differently. To analyze such a sensor layer we

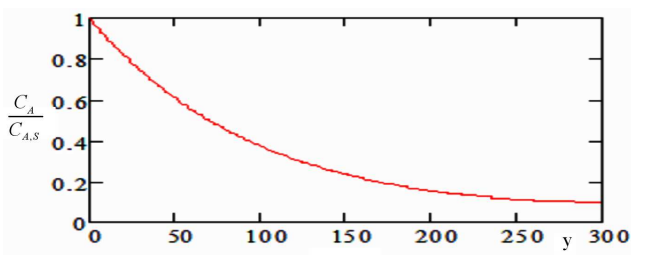

Fig. 3. Simulated gas concentration profiles inside a sensing film (thickness $300 \mathrm{~nm}$ ) for a fixed value of $\sqrt{k / D_{\mathrm{K}}}=0.01$ and fixed temperature.

assumed that the film is a uniform stack of infinitesimally thin sheets with a variable concentration of gas molecules and with a different electric conductance. Each sublayer is in another distance from the piezoelectric waveguide.

\section{Model of the sensor layer divided into $N$ sublayers}

The schematic diagram in Fig. 4 presents blocks whose common admittance was calculated basing on the law of impedance transformation and electronic equations. Then we applied the common impedance to the Ingebrigtsen formula. To the calculate surface conductance of the sublayers we used the profile of concentration of the gas. In the work we were focused on the box, which consists of the Ingebrigtsen formula, the transformation law and gas profile in the semiconducting layer. The main assumption is the additivity of the electric effect from each layer on the SAW propagation $[10,11]$. The perturbation in SAW velocity results from changes in the electric conductivity of the layer, $\sigma_{\mathrm{S}}$, due to the interaction of these chemically active layers with gas [12-15].

The source of perturbation for SAW can be each layer above the piezoelectric waveguide. The SAW propagation can be disturbed in any arbitrary plane above the 


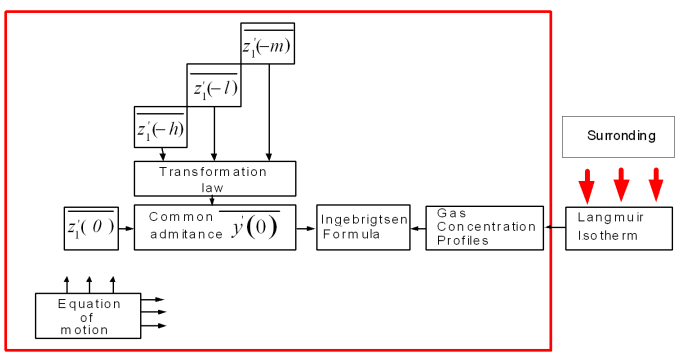

Fig. 4. Analytical diagram of the SAW gas sensor.

piezoelectric substrate. The case of interaction of an active sensing layer with gas at a distance $h, m$ and $l$ from the piezoelectric substrate is shown in Fig. 5.

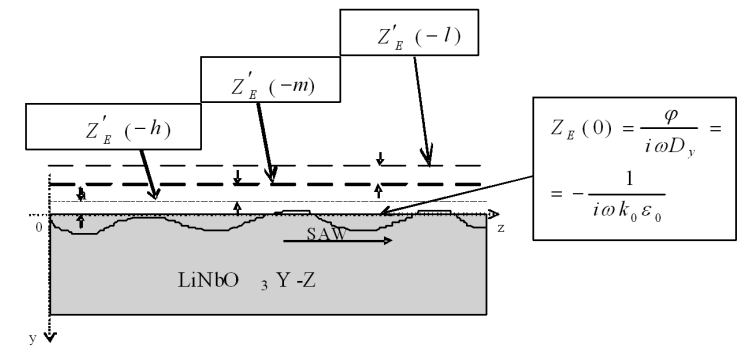

Fig. 5. Electrical waveguide surface perturbations by three sublayers.

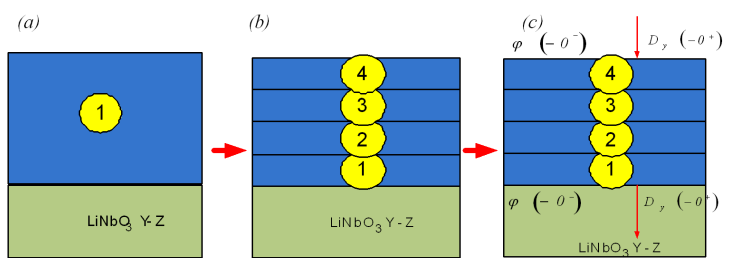

Fig. 6. Model of a sensor layer divided into $n$ sublayers.

Now the sensor layer was divided into $n$ sublayers. The first diagram (Fig. 6) presents a single layer of the semiconductor (a) on the piezoelectric substrate, on the next diagram (b) a semiconductor layer divided into four sublayers. In the last diagram (c) we see an equivalent model which has been prepared to apply the gas profiles, when gas will be adsorbed by the sensing layer. Let us consider that the semiconductor film contains $n$ sublayers with the thickness $d$ each. We get the following model shown in Fig. 7.

\section{Application of the transformation law into the Ingebrigtsen formula for a single layer}

The perturbation of a piezoelectric Rayleigh wave by any arbitrary electric impedance at the boundary close to the substrate surface has been described in this sec-

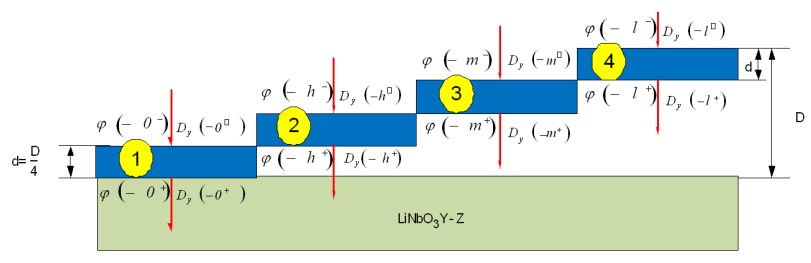

Fig. 7. Equivalent model of the sensor layer, divided into $n$ sublayers, which has been prepared to apply the gas profile.

tion. Figure 5 shows a typical illustration of this problem, which is relevant to calculations of the beat wavelength in the piezoelectric surface wave coupling. Let us apply the Ingebrigtsen formula

$$
\left(\frac{\Delta k}{k_{0}}\right) \cong-\left(\frac{\Delta v}{v_{0}}\right)_{\mathrm{SC}} \frac{1+\mathrm{i} z_{E}^{\prime}(0)}{1-\mathrm{i} \frac{\varepsilon_{p}^{\mathrm{T}}}{\varepsilon_{0}} z_{E}^{\prime}(0)},
$$

where $v_{0}$ is the velocity of the acoustic surface wave, $k_{0}$ is the propagation factor.

In this problem, the impedance at $y=-h$ is transformed into an equivalent impedance at the substrate surface $y=0[4,5]$ (Fig. 8). The transformation law, which corresponds to the impedance transformation along the electric transmission line, is derived by writing general expressions for $\varphi^{\prime}$ and $D_{y}^{\prime}$ in the region $0>y>-h$. Since $\varphi^{\prime}$ satisfies Laplace's equation $\left(\nabla^{2} \Phi=0\right)[16]$, these are

$$
\begin{aligned}
& \varphi^{\prime}=\left(A \mathrm{e}^{k_{0} y}+B \mathrm{e}^{-k_{0} y}\right) \mathrm{e}^{-k_{0} z}, \\
& D_{y}^{\prime}=-\varepsilon_{0} \frac{\partial \Phi^{\prime}}{\partial y}=-k_{0} \varepsilon_{0}\left(A \mathrm{e}^{k_{0} y}-B \mathrm{e}^{-k_{0} y}\right) \mathrm{e}^{k_{0} z},
\end{aligned}
$$

where the perturbed propagation constant $k_{0}^{\prime}$ has been approximated by unperturbed $k_{0}$. Normalized impedance on the surface $y=0$ is

$$
z_{E}^{\prime}(0)=\frac{Z_{E}^{\prime}(0)}{\left|Z_{E}(0)\right|}=-\mathrm{i} k_{0} \varepsilon_{0}\left(\frac{\varphi^{\prime}}{D_{y}^{\prime}}\right)_{y=0} .
$$

The normalized impedance at any arbitrary plain $y$ is therefore

$$
z_{E}^{\prime}(y)=\frac{-\mathrm{i} k_{0} \varepsilon_{0}\left(\mathrm{e}^{k_{0} y}+\frac{B}{A} \mathrm{e}^{-k_{0} y}\right) \mathrm{e}^{-k_{0} y}}{-k_{0} \varepsilon_{0}\left(\mathrm{e}^{k_{0} y}-\frac{B}{A} \mathrm{e}^{-k_{0} y}\right) \mathrm{e}^{-k_{0} y}} .
$$

The constant $B / A$ is evaluated by setting $y=-h$ in (14), and this gives the impedance transformation law

$$
z_{E}^{\prime}(0)=\frac{\mathrm{i} \tanh \left(k_{0} h\right)+z_{E}^{\prime}(-h)}{1-\mathrm{i} z_{E}^{\prime}(-h) \tanh \left(k_{0} h\right)} .
$$

When $h=0$, the transformation law takes the following form:

$$
z_{E}^{\prime}(0)=z_{E}^{\prime}(-h) \text {. }
$$

By using the transformation law we counted the common admittance "which is seen by an acoustoelectric wave on the plane $y=0$ ". We applied the common admittance in this way in the Ingebrigtsen formula, which consisted in the definition impedance $z_{E}^{\prime}(0)$ on the surface at $y=0$. The main problem was to calculate common normalized admittance which consists easy form to apply in the Ingebrigtsen formula. 


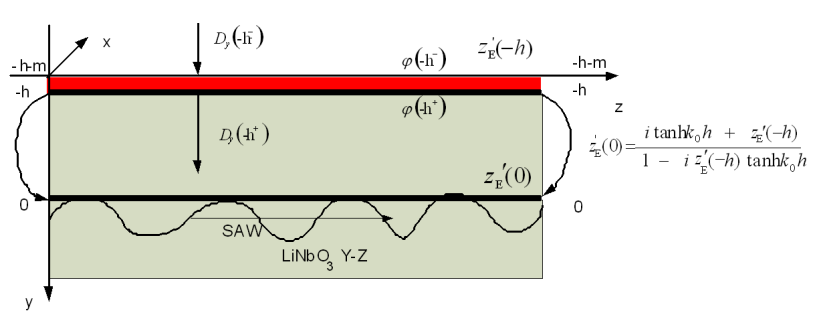

Fig. 8. The idea of the impedance transformation law.

Theoretical results calculated from (15) are compared with experimental data for SAW sensors [5].

\section{The Ingebrigtsen formula for single and multilayer structures of sensor layers}

The Ingebrigtsen formula for one layer in the case of the electrical effect (assuming that the mass of the layer $m=0)$ is

$$
\frac{\Delta v}{v_{0}}=-\operatorname{Re}\left(\frac{\Delta k}{k_{0}}\right)=-\frac{K^{2}}{2} \frac{\sigma_{0}^{2}}{\sigma_{0}^{2}+\left(v_{0} C_{\mathrm{S}}\right)^{2}} .
$$

The verification of the boundary conditions is

$$
\lim _{\sigma \rightarrow 0}\left(\frac{\Delta v}{v_{0}}\right)=0 \text { and } \lim _{\sigma \rightarrow \infty}\left(\frac{\Delta v}{v_{0}}\right)=-\frac{K^{2}}{2},
$$

which means that for $\sigma \rightarrow 0$ there is no interaction of the acoustic field with electrons in the sensor layer because there are no electrons and for $\sigma \rightarrow \infty$ no interaction of the acoustic field with electrons in sensor layer because of the screening effect.

The influence of the interaction between the electrical potential associated with the acoustic wave and the carriers of the electric charge in this layer leads to a decrease of the velocity. The Ingebrigtsen formula for $n$ sublayers, where, i.e. $y=h, l, m$ is as follows. By combining Eqs. (1), (15) with Eq. (10), followed by rearranging, one can obtain the following expression for the change of velocity vs. conductivity:

$$
\begin{aligned}
& f(y)=f\left(y_{1}, y_{2}, y_{3}, \ldots, n\right), \quad f(y)=f(h, m, l) \\
& \frac{\Delta v}{v_{0}}=-\operatorname{Re}\left(\frac{\Delta k}{k_{0}}\right)=-\frac{K^{2}}{2} \sigma_{\mathrm{CS}}^{2}\left[1+\sum_{i=1}^{n-1} f\left(y_{i}, \sigma_{\mathrm{CS}}\right)\right]^{2} \\
& /\left\{\sigma_{\mathrm{CS}}^{2}\left[1+\sum_{i=1}^{n-1} f\left(y_{i}, \sigma_{\mathrm{CS}}\right)\right]^{2}\right. \\
& \left.+\left[1+\sum_{i=1}^{n-1} g\left(y_{i}, \sigma_{\mathrm{CS}}\right)\right]^{2}\left(v_{0} C_{\mathrm{S}}\right)^{2}\right\},
\end{aligned}
$$

where $n$ - quantity of sublayers and $C_{\mathrm{S}}=\varepsilon_{0}+\varepsilon_{p}^{\mathrm{T}}$ :

$$
\begin{aligned}
f & \left(y_{i}, \sigma_{\mathrm{CS}}\right) \\
& =\sum_{i=1}^{n-1} \frac{1-[\tanh (k y)]^{2}}{[1+\tanh (k y)]^{2}+\left[\tanh (k y) \frac{\sigma_{\mathrm{CS}}}{\varepsilon_{0} v_{0}}\right]^{2}},
\end{aligned}
$$

$$
\begin{aligned}
g & \left(y_{i}, \sigma_{\mathrm{CS}}\right) \\
& =\sum_{i=1}^{n-1} \frac{[1+\tanh (k y)]^{2}+\tanh (k y)\left(\frac{\sigma_{\mathrm{CS}}}{\varepsilon_{0} v_{0}}\right)^{2}}{[1+\tanh (k y)]^{2}+\left[\tanh (k y) \frac{\sigma_{\mathrm{CS}}}{\varepsilon_{0} v_{0}}\right]^{2}} .
\end{aligned}
$$

Now we checked the boundary conditions in the formula for $n$ sublayers. When sublayers are placed in the plane $y=0$, we have the following circumstances:

$$
\begin{aligned}
& h, m, l \rightarrow 0, \\
& {\left[1+\sum_{i=1}^{n-1} f\left(y_{i}, \sigma_{\mathrm{CS}}\right)\right] \rightarrow n,} \\
& {\left[1+\sum_{i=1}^{n-1} g\left(y_{i}, \sigma_{\mathrm{CS}}\right)\right] \rightarrow n,} \\
& \frac{\Delta v}{v_{0}}=-\operatorname{Re}\left(\frac{\Delta k}{k_{0}}\right)=-\frac{K^{2}}{2} \frac{\left(n \sigma_{\mathrm{CS}}\right)^{2}}{\left(n \sigma_{\mathrm{CS}}\right)^{2}+\left(n v_{0} C_{\mathrm{S}}\right)^{2}}, \\
& \lim _{\sigma \rightarrow \infty}\left(\frac{\Delta v}{v_{0}}\right)=-\frac{K^{2}}{2} \text { and } \lim _{\sigma \rightarrow 0}\left(\frac{\Delta v}{v_{0}}\right)=0,
\end{aligned}
$$

where $\sigma_{\mathrm{CS}}$ is the surface conductivity of the sublayer with the thickness $d$.

\section{The Ingebrigtsen formula for multilayer structure with gas concentration profile}

Applying the concentration profile in the Ingebrigtsen formula we assumed

$$
\begin{aligned}
& \sigma_{\mathrm{CS}}=\sigma(y), \\
& \sigma(y)=\sigma_{0}\left[1+a C_{\mathrm{A}}(y)\right] .
\end{aligned}
$$

By combining Eqs. (27), (28) with Eq. (19) followed by rearranging, we obtained the following expression for the change of velocity vs. concentration of target gas outside the film in the surrounding:

$$
\begin{aligned}
& \frac{\Delta v}{v_{0}}=-\operatorname{Re}\left(\frac{\Delta k}{k_{0}}\right)=-\frac{K^{2}}{2} \sigma_{0}^{2}\left(1+a C_{\mathrm{A}}\right)^{2} \\
& \times\left[1+\sum_{i=1}^{n-1} f\left(y_{i}, \sigma\left(y_{i}\right)\right)\right]^{2} \\
& /\left\{\sigma_{0}^{2}\left(1+a C_{\mathrm{A}}\right)^{2}\left[1+\sum_{i=1}^{n-1} f\left(y_{i}, \sigma\left(y_{i}\right)\right)\right]^{2}\right. \\
& \left.+\left[1+\sum_{i=1}^{n-1} g\left(y_{i}, \sigma\left(y_{i}\right)\right)\right]^{2}\left(v_{0} C_{\mathrm{S}}\right)^{2}\right\}, \\
& \sigma_{T_{2}}=\sigma_{T_{1}} \exp \left(\frac{E_{g}}{2 k} \frac{T_{2}-T_{1}}{T_{1} T_{2}}\right),
\end{aligned}
$$

where $T_{1}=300 \mathrm{~K}, \sigma_{T_{1}}=\sigma_{T_{1}}(300 \mathrm{~K})=\sigma_{0}$

By combining Eqs. (2), (8), (30) with Eq. (29), followed by rearranging, we obtained the following expression for the change of velocity vs. temperature of target gas outside the film in the surrounding and changing the "point of work" sensor vs. temperature:

$$
\frac{\Delta v}{v_{0}}=-\operatorname{Re}\left(\frac{\Delta k}{k_{0}}\right)=-\frac{K^{2}}{2} \sigma_{T_{2}}^{2}\left(1+a C_{\mathrm{A}}\right)^{2}
$$




$$
\begin{aligned}
& \times\left[1+\sum_{i=1}^{n-1} f\left(y_{i}, \sigma\left(y_{i}\right)\right)\right]^{2} \\
& /\left\{\sigma_{T_{2}}^{2}\left(1+a C_{\mathrm{A}}\right)^{2}\left[1+\sum_{i=1}^{n-1} f\left(y_{i}, \sigma_{T_{2}}\right)\right]^{2}\right. \\
& \left.+\left[1+\sum_{i=1}^{n-1} g\left(y_{i}, \sigma\left(y_{i}\right)\right)\right]^{2}\left(v_{0} C_{\mathrm{S}}\right)^{2}\right\}
\end{aligned}
$$

Equation (31) describes the change of velocity dependent on the change of temperature.

We verified the Ingebrigtsen formula with a gas profile. When the concentration profile disappeared and sublayers are placed in the plane $y=0$ we have

$$
\begin{aligned}
& h \rightarrow 0, \quad l \rightarrow 0, \quad m \rightarrow 0, \quad C_{\mathrm{AS}} \rightarrow 0, \\
& {\left[1+\sum_{i=1}^{n-1} f\left(y_{i}, \sigma_{\mathrm{CS}}\right)\right] \rightarrow n,} \\
& {\left[1+\sum_{i=1}^{n-1} g\left(y_{i}, \sigma_{\mathrm{CS}}\right)\right] \rightarrow n .}
\end{aligned}
$$

When we checked the boundary conditions in the formula for $n$ sublayers with a gas profile we obtained the following formula for a single layer without target gas:

$$
\frac{\Delta v}{v_{0}}=-\operatorname{Re}\left(\frac{\Delta k}{k_{0}}\right)=-\frac{K^{2}}{2} \frac{\left(\sigma_{0}\right)^{2}}{\left(\sigma_{0}\right)^{2}+\left(v_{0} C_{\mathrm{S}}\right)^{2}} .
$$

The verification of the boundary conditions for the Ingebrigtsen formula with the concentration gas profile renders correct results, when the following data reach extreme values.

\section{Conclusions}

a) This paper presents the theoretical basis of SAW gas sensors operating with a semiconducting thin film as a chemical sensor layer with the concentration gas profile.

b) The concentration profiles of target gas inside the film can be estimated as a function of Knudsen's diffusion coefficient, $D_{\mathrm{K}}$, first-order reaction rate constants, $k$, and thickness of the sensor layer, $d$.

c) Surface conductivity $\sigma(y)$ of a thin film at a fixed temperature $T$ can be derived from the gas concentration profile by introducing electrical conductance of the sheet which is assumed to be linear to the gas concentration. The linearity constant, $a$, is defined as sensitivity coefficient.

d) We can use profile concentration in order to point out the response of the SAW gas sensor and divide the semiconductor layer into $n$ sublayers. When the transformation law is applied we calculated the impedance on the surface $y=0$.

e) In SAW sensors there may occur two effects: electrical (acoustoelectric) and mass effect. In this paper we considered the electrical effect as perturbations in electrical boundary conditions. Perturbations in electrical boundary conditions are important in a sensor with a conducting sensor layer. These effects are additive because of their small values.

\section{Acknowledgments}

This work is financed as the grant of Ministry of Science and Higher Education No. N N505 374237.

\section{References}

[1] G. Sakai, N. Matsunaga, E. Shimanoe, N. Yamazone, Sensors Actuators B 80, 125 (2001).

[2] D.C. Malocha, B.H. Fischer, IEEE Trans. Ultrason. Ferroelectr. Freq. Control 57, 163 (2010).

[3] D.C. Malocha, B.H. Fischer, in: School of Electrical Engineering \& Computer Science, University of Central Florida, Orlando (USA) 2009, p. 32816.

[4] T. Hejczyk, M. Urbańczyk, W. Jakubik, Acta Phys. Pol. A 118, 1153 (2010).

[5] B.A. Auld, Acoustic Fields and Waves, Vol. 2, Wiley, New York 1973.

[6] J.W. Gardner, Sensors Actuators B 1, 166 (1990).

[7] T. Pisarkiewicz, Gas Microsensors, AGH, Kraków 2007 (in Polish).

[8] D.E. Williams, A. Hilger, Solid State Gas Sensors, Academic Press, Bristol 1987.

[9] T. Pustelny, A. Opilski, B. Pustelny, Acta Phys. Pol. A 114, A-181 (2009).

[10] W. Jakubik, M. Urbańczyk, Arch. Acoust. 22, 187 (1997).

[11] W. Jakubik, Thin Solid Films 515, 8345 (2007).

[12] W. Jakubik, Sensors Actuat. B 96, 321 (2003).

[13] W. Jakubik, M. Urbańczyk, E. Maciak, T. Pustelny, Acta Phys. Pol. A 116, 315 (2009).

[14] B. Pustelny, T. Pustelny, Acta Phys. Pol. A 116, 383 (2009).

[15] W. Jakubik, M. Urbańczyk, J. Techn. Phys. 38, 589 (1997).

[16] G.S. Kino, IEEE Trans. Electron Dev. ED-18, 347 (1971). 